\title{
Purpose, use, and preparation of clinical practice guidelines for the management of biliary tract and ampullary carcinomas
}

\author{
Tadahiro Takada ${ }^{1}$, Masaru Miyazaki ${ }^{2}$, Shuichi Miyakawa ${ }^{3}$, Kazuhiro Tsukada $^{4}$, Masato Nagino ${ }^{5}$, \\ SAtoshi Kondo ${ }^{6}$, JunjI Furuse ${ }^{7}$, Hiroya SAito ${ }^{8}$, TOShio Tsuyuguchi ${ }^{9}$, Fumio Kimura ${ }^{2}$, Hideyuki Yoshitomi ${ }^{2}$, \\ Satoshi Nozawa ${ }^{2}$, Masahiro Yoshida ${ }^{1}$, Keita Wada ${ }^{1}$, Hodaka Amano ${ }^{1}$, and Fumihiko Miura ${ }^{1}$ \\ ${ }^{1}$ Department of Surgery, Teikyo University School of Medicine, 2-11-1 Kaga, Itabashi-ku, Tokyo, 173-8605, Japan \\ ${ }^{2}$ Department of General Surgery, Chiba University Graduate School of Medicine, Chiba, Japan \\ ${ }^{3}$ Department of Gastroenterological Surgery, Fujita Health University, Toyoake, Japan \\ ${ }^{4}$ Department of Surgery and Science, Graduate School of Medicine and Pharmaceutical Science for Research, University of Toyama, \\ Toyama, Japan \\ ${ }^{5}$ Division of Surgical Oncology, Department of Surgery, Nagoya University Graduate School of Medicine, Nagoya, Japan \\ ${ }^{6}$ Department of Surgical Oncology, Hokkaido University Graduate School of Medicine, Sapporo, Japan \\ ${ }^{7}$ Hepatobiliary and Pancreatic Oncology Division, National Cancer Center Hospital East, Chiba, Japan \\ ${ }^{8}$ Department of Radiology, Asahikawa Kosei General Hospital, Asahikawa, Japan \\ ${ }^{9}$ Department of Medicine and Clinical Oncology, Chiba University Graduate School of Medicine, Chiba, Japan
}

\begin{abstract}
Apart from periampullary carcinoma, the prognosis of biliary tract carcinomas, including hilar cholangiocarcinoma, extrahepatic biliary tract carcinoma, and gallbladder carcinoma, remains poor. Sophisticated diagnostic skills and treatment methods and their application are naturally required to achieve better treatment results for biliary tract carcinomas. However, it is not too much to say that, due to the paucity of high-level evidence for the management of these carcinomas, medical care by healthcare providers in clinics and at medical institutes throughout the world is currently delivered without common consensus and common standards. The clinical practice guidelines for the management of biliary carcinoma outlined here were produced with the aim that they could be used by physicians involved in the care of biliary tract carcinomas, as indicators that could help them provide their patients with the most appropriate care possible at this time. Also, the guidelines were prepared to provide measures that could assure patients with biliary tract carcinomas of safe medical care. The present guidelines are characterized by their clarification of clinical questions assumed to be often shared by healthcare professionals. For clarity, we divided the contents of the guidelines into eight areas. In each area, clinical questions are presented, together with recommendations of clinical actions in response to the question. As mentioned already, there is a paucity of high-level evidence in this area; therefore, the recommendations are classified into grades, of which there are five: A, strongly recommend performing the clinical action; $\mathrm{B}$, recommend performing the clinical action; $\mathrm{C} 1$, the clinical action may be useful, although there is a lack of high-level scientific evidence; $\mathrm{C} 2$, clinical action not definitively recommended because of insufficient scientific evidence; D, recommend not performing the clinical action. The grading of the recommendations is based on the determination of the level
\end{abstract}

Offprint requests to: T. Takada

Received: October 1, 2007 / Accepted: October 22, 2007 of evidence in references on which the recommendation is based.

Key words Guidelines · Biliary · Hilar cholangiocarcinoma · Gallbladder carcinoma

\section{Purpose of the clinical practice guidelines for the management of biliary tract and ampullary carcinomas (the guidelines)}

No guidelines for the diagnosis and treatment of biliary tract carcinoma, the prognosis of which remains poor, have been published to date, although a set of guidelines for intrahepatic cholangiocarcinoma was published in 2002 by Khan et al. ${ }^{1}$ There remain many problems to be solved concerning the diagnosis and treatment of biliary tract carcinoma so that better treatment results can be achieved for this disease. We (The Japanese Society of Hepato-Biliary-Pancreatic Surgery [JSHBPS]) produced the present guidelines, recognizing that a high level of expertise is required for those concerned with the diagnosis and treatment of the disease. The greatest obstacle encountered most frequently in preparing the present guidelines was the paucity of high-level evidence for the diagnosis and treatment of biliary tract carcinoma and the lack of practical guidelines for coping with this problem. Currently, under these circumstances, there are wide disparities in the levels of diagnosis and treatment among different institutions. However, in assessing the strength of recommendations for treatment, because of the paucity of high-level evidence in this field, the members of the Organizing Committee formed for creating the guidelines focused on building a concensus. Occasionally, decisions were subject to modifications on 
the basis of discussions at an open forum. The aim of the guidelines was to supply indicators to physicians involved in the care of this disease, to provide their patients with the safest and most appropriate care.

\section{Notes on the use of the guidelines}

The entire contents of the present guidelines were endorsed upon discussions held among all the members of the Organizing Committee and the Publishing Committee. The guidelines consist of algorithms that outline diagnosis and treatment, followed by clinical questions. In view of the fact that there is still a wide variation in treatment policies among medical institutions, the presentation of the contents is made in the form of clinical questions. ${ }^{2,3}$ The contents are divided into eight areas to elucidate the clinical questions considered to be shared by healthcare professionals and to clarify the guidelines with respect to these questions at this stage of publication. The questions in each area are presented under the heading of "clinical questions." The presence or absence of recommendations for a clinical action, the strength of the recommendations, and statements on how recommendations are made are provided with each question. Recommendations and statements are formulated on the basis of evidence. The strength of treatment recommendations in response to each question was determined in accordance with the following policy. Compared with breast cancer and gastric cancer, only a limited amount of high-level evidence has been obtained from randomized controlled trials for the diagnosis and treatment of biliary tract carcinoma. As a result, we aimed to reach a consensus on the grading of the strength of recommendations. This was done and the strength of recommendations was stated according to five grades:

A, Strongly recommend performing the clinical action.

$\mathrm{B}$, Recommend performing the clinical action.

$\mathrm{C} 1$, Clinical action may be useful although there is a lack of high-level scientific evidence.

$\mathrm{C} 2$, Not definitively recommended, because of insufficient scientific evidence.

$\mathrm{D}$, Recommend not performing the clinical action.

\section{Perspective on "guidelines"}

With regard to the present guidelines, from the perspective of "guidelines," they represent the most standard clinical and practical indicators at this time. However, they should not be coercive for the practical management of individual patients. Final decisions on management should be made on the basis of conditions at individual institutions (depending on personnel, experience, instruments, etc.) and the individual characteristics of patients.
The Japanese Society of Hepato-Biliary-Pancreatic Surgery (JSHBPS) is responsible for the statements in the present guidelines. The JSHBPS and the Organizing Committee members are not liable for any consequences arising from any treatment, for which individual physicians involved in the treatment should be responsible. The dosages described in the text are for adult patients.

\section{How the guidelines were formulated}

With evidence-based medicine (EBM) as the core concept, the first Preparation Committee for "the guidelines for the management of biliary tract carcinoma" was held on May 12, 2005, to discuss how to formulate the guidelines. The guidelines were prepared according to the following procedures: (1) Comprehensive search using key words for relevant literature on biliary tract carcinoma in the past 20 years. (2) Assessment and classification of the abstracts of all retrieved articles, on the basis of contents, by at least two reviewers, and selection of literature considered useful. (3) Using the selected literature as reference matter, each committee member prepared a draft of clinical questions in the area in which he or she was involved. (4) From all the questions selected by all committee members, clinical questions considered appropriate were selected at the general meeting. (5) Preparation of statements on clinical questions of each specialty. (6) Review of the presence/absence of recommendations, strength of recommendations and statements proposed at the general meeting, and checking of the contents of statements. (7) Check of the final draft by all committee members. (8) Assessment by the members of the Review Committee of the draft prepared according to the above procedures. (9) A public hearing was held to hear opinions from an audience with wide medical specialties. (10) New committee members of the Publishing Committee were elected from the Organizing Committee to further review the final draft. So that the draft would be objective and evidence-based, it was further checked for consistency in the strength of recommendations and statements of clinical questions. The statements were modified to be more readable. Thus, the final draft was prepared.

\section{Comprehensive literature search}

References written in English were searched for on MEDLINE in June 2005. Reference formulas used and the number of items retrieved are shown below. Systematic review articles were also searched for using the formulas below. References were further searched for, where necessary, by means of a manual search. 
Search for English-language references

1. Biliary tract neoplasms [MeSH] limits: publication date from 1985, English, humans.

2. Publication types: Clinical trial or Meta-analysis or Practice Guideline or Randomized Controlled Trial or Review - 1266 items.

\section{Systematic reviews}

Cochrane library: key word "biliary" 59 items.

MEDLINE:

Search 1. "biliary tract neoplasms" [MeSH] limits: only items with abstracts, English, publication date from 1985 to 2006, Clinical Trial, Meta-analysis, Randomized Controlled Trial, Review - 1136 items

Search 2. "biliary tract neoplasms" $[\mathrm{MeSH}]$ or "gallbladder cancer" or "common bile duct cancer" limits: English, publication date from 1985 to 2006, humans - 491 items

Search 3. $((()((()((" M e t a-A n a l y s i s " ~[M e S H$ terms] or meta-analysis[pt]) or medline [tiab]) or ((( (metaanalyses [tiab] or metaanalysis[tiab]) or metaanalytic[tiab]) or metaanalytical [tiab]) or metaanalytically [tiab])) or "metaanalysis" [All Fields]) or (((( overview [tiab] or overview/literature [tiab]) or overviewed [tiab]) or overviewer [tiab]) or overviewing [tiab]) or overviews [tiab])) or clinical trial [pt]) or multicenter study[pt]) or evaluation studies[pt]) or validation studies [pt]) or review [pt]) or (systematic review [All Fields] or systematic reviews [All Fields])) - 1827246 items

Search 2 and Search 3, but not Search 1, - 314 items

\section{Final selection of appropriate references by Publishing} Committee members

On the basis of an evaluation by at least two Publishing Committee members of the importance of abstracts of selected references, 669 English-language references were finally selected.

Preparation of drafts of clinical questions

Using the literature thus selected as references, drafts of clinical questions were prepared by the responsible Publishing Committee members, and 227 questions in total were proposed.
Final selection of clinical questions from the drafts At a general meeting held on July 10, 2006, the decision was made that 33 clinical questions should be adopted for each of the eight specialized areas.

\section{Preparation of statements for clinical questions}

The statements of clinical questions and introductions were prepared for each specialized area by the responsible Publishing Committee members. Another general meeting was held and the number of clinical questions to be adopted was changed to 35 .

The strength of recommendations for individual clinical questions was given in related statements. Determination of the level of evidence for individual references, on which the strength of recommendations was determined, was made according to the criteria shown in Tables 1 and 2. In view of the lack of high-level evidence on the treatment of biliary tract carcinoma, the decisions on treatment policies were made by taking into account the findings collected from case studies.

\section{Strength of recommendations and evaluation of evidence levels (Tables 1 and 2)}

The "grade of recommendations" was discussed many times at an Organizing Committee meeting and at a second public hearing meeting. There are many criteria for the strength of recommendations in many clinical practice guidelines, and there are no worldwide standards. After all the discussions, we were able to reach the following consensus on the grading of the strength

Table 1. Strength of recommendations

Table 2. Levels of evidence

Level I

Level II

Level III

Level IV

Level V

Level VI
Systematic review/meta-analysis

One or more randomized clinical trials

Nonrandomized controlled trials

Analytic epidemiology (cohort studies and case-control studies)

Descriptive study (case reports and case-series studies)

Opinions of expert panels and individual experts not based on patient's data 
of recommendations. The aim of the grading of recommendations is to present indicators which will help physicians involved in the care of patients with biliary tract carcinoma to provide their patients with the safest and most appropriate care.

\section{Decisions on clinical questions and review of algorithms}

The statements for the clinical questions were modified by the responsible Publishing Committee members. A further general meeting was held to review all the clinical questions. The final number of questions was changed to 36. Diagnosis and treatment algorithms were also prepared.

\section{Evaluation by Review Committee members}

Evaluations and suggestions for the final draft were made by the External Review Committee.

Additions and modifications were made after discussions by the Publishing Committee members.

\section{Public hearing}

At two public hearings (the first on June 7, 2007; the $19^{\text {th }}$ Annual Meeting of the JSHPBS, and the second, on September 29, 2007; the $43^{\text {rd }}$ Annual Meeting of Japan Biliary Association), comments were requested from the audience, particularly from physicians from a wide area of medical specialties, and then the final corrections of the draft were made.

\section{Publishing Committee}

The Publishing Committee was organized after the first public hearing. Reexaminations, additions, and corrections were made with respect to all the expressions, sentences, and terminology, etc., by all the Publishing Committee members. For the contents, repeated discussions were held until a sufficient consensus was achieved.

The final version of the final draft was made public at the second public hearing, and comments from the general audience were requested. The final version was also evaluated by the External Review Committee members. The draft was finalized taking into account these comments and the evaluation.

\section{Diffusion of the guidelines}

The guidelines, Evidence-based clinical practice guidelines for the management of biliary tract carcinoma, were published in Japanese by Igaku-Tosyo Syuppan.

The guidelines were posted on the Internet on the JSHBPS home page, Japan Council for Quality Health Care; and, on the medical information network distribution service (Minds) home page.

\section{Plan for publication of the revised edition}

Evaluation and accumulation of pertinent evidence is being continued by the Organizing Committee of the guidelines, which has been established permanently at the JSHBPS. A revised edition is expected to be published every 4 years, but the timing of the revisions will be subject to change depending upon advances in medical science and care.

\section{Funding}

Finance for the preparation of the present guidelines was provided under the auspices of a research subsidy for fiscal 2005 and 2006 (Integrated Research Project for Assessing Medical Safety and Medical Technology, sponsored by the Japanese Ministry of Health, Labour, and Welfare, and the JSHBPS).

Acknowledgment. We would like to express our deep gratitude to the members of the the Japanese Association of Biliary Surgery, the Japanese Society of HepatoBiliary-Pancreatic Surgery, and the Japan Society of Clinical Oncology, who provided us with great support and guidance in the preparation of the Guidelines. This process was conducted as part of the Integrated Research Project for Assessing Medical Technology 2005 and 2006 sponsored by the Japanese Ministry of Health, Labour, and Welfare.

We truly appreciate the following active working members who developed the draft of the evidencebased clinical practice Guidelines for the treatment of biliary tract cancer (Japanese version, 2007): Masahiro Kai (Miyazaki), Yasutoshi Kimura (Sapporo), Shigeaki Sawada (Toyama), Hiroaki Shimizu (Chiba), Hisatoshi Nakagawara (Kanazawa), Kohei Nakachi (Kashiwa), and Hiroyuki Yoshitome (Chiba). We also appreciate very much the following members who reviewed and approved the final Japanese version of the guidelines: Hiromitsu Saisyo (Ichikawa), Munemasa Ryu (Chiba), Satoru Shikata (Kyoto), and Yuji Nimura (Nagoya). 
T. Takada et al: Background of guidelines for biliary cancer

\section{References}

1. Khan SA, Davidson BR, Goldin R, Pereira SP, Rosenberg WM, Taylor-Robinson SD, et al.; British Society of Gastroenterology. Guidelines for the diagnosis and treatment of cholangiocarcinoma: consensus document. Gut 2002;51:VI1-9.

2. Takada T, Hirata K, Kawarada Y, Mayumi T, Yoshida M, Sekimoto M, et al. JPN Guidelines for the management of acute pancreatitis: cutting-edge information. J Hepatobiliary Pancreat Surg 2006;13: 2-6.

3. Takada T, Kawarada Y, Nimura Y, Yoshida M, Mayumi T, Sekimoto $\mathrm{M}$, et al. Background: Tokyo guidelines for the management of acute cholangitis and cholecystitis. J Hepatobiliary Pancreat Surg 2007;14:1-10. 